\title{
A NEW PROOF OF THE TRANSPOSITION THEOREM
}

\author{
LEOPOLD FLATTO
}

It is well known that the minimax theorem can be deduced from various forms of the transposition theorem (see e.g. [2] and [3]). In particular it follows from the following:

Transposition Theorem. Let $A$ be a real $m$ by $n$ matrix and $A^{\prime}$ its transpose; let $x$ and $y$ denote respectively $n$ - and $m$-dimensional real column vectors. Then either $A x \geqq 0$ for some $x \geqq 0, x \neq 0$ or $A^{\prime} y \leqq 0$ for some $y \geqq 0, y \neq 0\left(x=\left(x_{1}, \cdots, x_{n}\right) \geqq 0\right.$ means $\left.x_{i} \geqq 0,0 \leqq i \leqq n\right)$.

It is shown in [3] that the above theorem follows readily from

Stiemke's Theorem [4]. If $S$ is a subspace of $R^{n}$ and $S^{+}$the orthogonal complement of, then $S \cup S^{+}$contains some vector $x \geqq 0, x \neq 0$.

In this note we obtain a formula for the number of orthants intersected by a subspace of $R^{n}$. Stiemke's theorem and ipso the above mentioned transposition theorem will be obtained as a direct consequence of the formula. We employ the following terminology. The hyperplanes $H_{1}, \cdots, H_{s}$ of $R^{n}(s \geqq n)$ are said to be in general position if the intersection of any $n$ of them is 0 . The $k$-dimensional subspace $S$ of $R^{n}$ is said to be in general position if the $n$ subspaces $H_{i} \cap S$, where $H_{i}=\left\{x \mid x_{i}=0\right\} \quad(1 \leqq i \leqq n)$, are hyperplanes of $S$ in general position. Letting $H_{i_{1}^{\prime} \cdots, i_{k}}=\left\{x \mid x_{i_{1}}=x_{i_{2}}=\cdots=x_{i_{h}}=0\right\}$ where $1 \leqq i_{1}<i_{2}<\cdots<i_{k} \leqq n$, this condition means that $S \cap H_{i_{1}}$ $\cdots \cap H_{i_{k}}=0$ or equivalently, $R^{n}=S \oplus H_{i_{1}}, \cdots, i_{k}$ for all choices of $1 \leqq i_{1}<i_{2}<\cdots<i_{k} \leqq n$. If the $k$ vectors $s_{1}=\left(s_{i 1}, \cdots, s_{i n}\right)$ $(1 \leqq i \leqq k)$ form a basis for $S$, then it is easily checked that $S$ is in general position if and only if all $k$ by $k$ minors of $\left(s_{i j}\right)(1 \leqq i \leqq k$, $1 \leqq j \leqq n)$ are $\neq 0$. An orthant of $R^{n}$ is defined to be the set $\left\{x \mid \epsilon_{i} x_{i}>0\right.$, $1 \leqq i \leqq n\}$ where $\left\{\epsilon_{i}\right\}$ denotes any fixed choice of \pm 1 's. We now prove the following:

Theorem. Let $S$ be a k-dimensional subspace of $R^{n}$ which is in general position. The number of orthants intersected by $S$ is

$$
2 \sum_{i=0}^{k-1}\left(\begin{array}{c}
n-1 \\
i
\end{array}\right)
$$

Received by the editors May 6, 1968. 
Proof. For $k=1$ and $n=k$, the result is obvious so that we may assume $2 \leqq k \leqq n$. Let $0_{n, k}=$ number of orthants intersected by $S$ $(2 \leqq k<n)$. Let $R_{n, k}=$ number of regions into which $n$ hyperplanes of $R^{k}$ in general position decompose $R^{k}(n \geqq 1, k \geqq 2)$. The orthants of $R^{n}$ intersected by $S$ stand in 1-1 correspondence with the regions into which the hyperplanes $H_{1} \cap S(1 \leqq i \leqq n)$ decompose $S$. Identifying $S$ with $R^{k}$ we obtain $0_{n, k}=R_{n, k}(2 \leqq k<n)$. The formula for $R_{n, k}$ is well known and can be found in [1]. We give another derivation of this formula.

Let $L_{1}, \cdots, L_{n+1}$ be $n+1$ hyperplanes of $R^{k}$ in general position decomposing $R^{k}(k \geqq 3)$ into $R_{n+1, k} k$-dimensional regions. Let $L_{n+1}$ intersect $c$ of these. Each of the regions is divided by $L_{n+1}$ into two regions so that $R_{n+1, k}=R_{n, k}+c$. Let $L_{i}^{\prime}=L_{i} \cap L_{n+1}(1 \leqq i \leqq n)$. Then $L_{1}^{\prime}, \cdots, L_{n}^{\prime}$ are hyperplanes of $L_{n+1}$ in general position decomposing $L_{n+1}$ into $R_{n, k-1}(k-1)$-dimensional regions. These regions are in 1-1 correspondence with the $c k$-dimensional ones intersected by $L_{n+1}$. Thus:

$$
R_{n+1, k}=R_{n, k}+R_{n, k-1} \quad(k \geqq 3, n \geqq 1) .
$$

Let $R_{k}(z)=\sum_{n=1}^{\infty} R_{n, k} z^{n}$. Since $R_{1, k}=2$ we conclude from (1) that

$$
R_{k}(z)=R_{k-1}(z)(z /(1-z))+2 z /(1-z) \quad(k \geqq 3) .
$$

Since $R_{n, 2}=2 n$ we have $R_{2}(2)=2 z /(1-z)^{2}=2\left[z /(1-z)+z^{2} /(1-z)^{2}\right]$. Repeated use of (2) yields

$$
R_{k}(z)=2\left[z /(1-z)+\cdots+z^{k} /(1-z)^{k}\right],
$$

Equating the $n$th coefficients of both sides of (3), we obtain the desired formula

$$
R_{n, k}=2 \sum_{i=0}^{k-1}\left(\begin{array}{c}
n-1 \\
i
\end{array}\right)
$$

We now obtain Stiemke's theorem as a consequence of the above formula. We may assume that $S$ is in general position; the case where $S$ is not in general position is then treated by a standard limiting argument.

Let $1 \leqq i_{1}<\cdots<i_{k} \leqq n, 1 \leqq j_{1}<\cdots<j_{n-k} \leqq n$ denote $n$ integers comprising all integers from 1 to $n . S \oplus H_{i_{1} \cdots i_{k}}=R^{n}$ as $S$ is in general position. Hence $0=\left(S \oplus H_{i_{1} \cdots i_{k}}\right)^{\perp}=S^{\perp} \cap H_{i_{1}}^{\perp} \cdots i_{k}=S^{\perp} \cap H_{j_{1} \cdots j_{n-k}}$. Since $S^{\perp} \cap H_{j_{1} \cdots j_{n-k}}=0$ for all choices of $1 \leqq j<\cdots<j_{n-k} \leqq n$, we conclude that $S^{\perp}$ is in general position. Thus the number of orthants intersected by $S^{\perp}$ is $2 \sum_{i=0}^{n-1-k}\left(\begin{array}{c}n-1 \\ i\end{array}\right)$. If $x, y$ are in the same orthant then $x \cdot y>0$ so that the orthants intersected by $S$ are distinct from those 
intersected by $S^{\perp}$. Since there are $2^{n}$ orthants and

$$
2 \sum_{i=0}^{k}\left(\begin{array}{c}
n-1 \\
i
\end{array}\right)+2 \sum_{i=k}^{n-1}\left(\begin{array}{c}
n-1 \\
i
\end{array}\right)=2 \sum_{i=0}^{n-1}\left(\begin{array}{c}
n-1 \\
i
\end{array}\right)=2^{n}
$$

we conclude that every orthant is intersected by $S$ or $S^{\perp}$. In particular this holds for the positive orthant, thus proving Stiemke's theorem.

\section{REFERENCES}

1. T. S. Motzkin, The probability of solvability of linear inequalities, Second Symposium on Linear Programming, Washington, D. C., 1955, vol. 2, pp. 607-611.

2. - Two consequences from the transposition theorem on linear inequalities, Econometrica 19 (1951), 184-185.

3. D. J. Newman, Another proof of the minimax theorem, Proc. Amer. Math. Soc. 11 (1960), 692-693.

4. A. W. Tucker, Extensions of theorems of Farkas and Stiemke, Abstract 76, Bull. Amer. Math. Soc. 56 (1950), 57.

Belfer Graduate School of Science, Yeshiva University 